\title{
Fractal Information by Means of Harmonic Mappings and Some Physical Implications
}

\author{
Maricel Agop ${ }^{1}$, Alina Gavriluţ ${ }^{2, *}$, Viorel Puiu Păun ${ }^{3}$, Dumitru Filipeanu 4 , \\ Florin Alexandru Luca ${ }^{4}$, Constantin Grecea ${ }^{5}$ and Liliana Topliceanu ${ }^{6}$ \\ 1 Department of Physics, Gheorghe Asachi Technical University of Iaşi, Iaşi 700506, Romania; \\ m.agop@yahoo.com \\ 2 Faculty of Mathematics, Alexandru Ioan Cuza (Al.I. Cuza) University, Iaşi 700506, Romania \\ 3 Department of Physics, Faculty of Applied Sciences, Politehnica University of Bucharest, Bucharest 060042, \\ Romania; paun@physics.pub.ro \\ 4 Economic and Marketing Department, Technical University Gheorghe Asachi from Iaşi, Iaşi 700050, \\ Romania; filipeanu@yahoo.com (D.F.); luca@yahoo.com (F.A.L.) \\ 5 Faculty of Physics, Alexandru Ioan Cuza (Al.I. Cuza) University, Iaşi 700506, Romania; grecea@yahoo.com \\ 6 Faculty of Engineering, University "Vasile Alecsandri" of Bacău, Bacău 600115, Romania; \\ topliceanu@yahoo.com \\ * Correspondence: gavrilut@uaic.ro; Tel.: +40-751-242-004
}

Academic Editor: Carlo Cattani

Received: 25 March 2016; Accepted: 20 April 2016; Published: 26 April 2016

\begin{abstract}
Considering that the motions of the complex system structural units take place on continuous, but non-differentiable curves, in the frame of the extended scale relativity model (in its Schrödinger-type variant), it is proven that the imaginary part of a scalar potential of velocities can be correlated with the fractal information and, implicitly, with a tensor of "tensions", which is fundamental in the construction of the constitutive laws of material. In this way, a specific differential geometry based on a Poincaré-type metric of the Lobachevsky plane (which is invariant to the homographic group of transformations) and also a specific variational principle (whose field equations represent an harmonic map from the usual space into the Lobachevsky plane) are generated. Moreover, fractal information (which is made explicit at any scale resolution) is produced, so that the field variables define a gravitational field. This latter situation is specific to a variational principle in the sense of Matzner-Misner and to certain Ernst-type field equations, the fractal information being contained in the material structure and, thus, in its own space associated with it.
\end{abstract}

Keywords: fractal information; fractal entropy; variational principle; mapping; economy; econometrics; management; economic statistics

\section{Introduction}

Complex systems are a very favorable medium for the appearance of instabilities [1,2]. These instabilities imply both chaos through different routes (intermittencies, quasi-periodicity, cascade of period-doubling bifurcations, sub-harmonic bifurcations, torus breakdown) and self-structuring through the generation of complex structures [3-5]. According to the classical concepts, all theoretical complex systems models (fluid models, kinetic models, etc.) assume that the dynamics of the complex system structural units occur on continuous and differentiable curves, so that they can be described in terms of continuous and differentiable motion variables (energy, momentum, density, etc.). These motion variables are exclusively dependent on the spatial coordinates and time. In reality, the complex systems dynamics proves to be much more complex, and the above simplifications cannot be expected to explain all of the aspects of the complex systems dynamics. However, this situation can still be standardized if we consider that the complexity of complex system interaction processes 
imposes different time resolution scales, while the evolution complex systems patterns lead to different degrees of freedom.

From the above-mentioned arguments, it results that the explanation of the complex systems dynamics can be based on the assumption that the motions of the complex system structural units take place on continuous, but non-differentiable curves (fractal curves), since, according to the procedures [6-9], only a fractal curve is dependent on the scale resolution. Moreover, according to the methodology from [10], through the dynamics of special topologies, which can implement evolution patterns in complex systems, it can lead to various degrees of freedom. Such an assumption can be sustained by a typical example, related to the collision processes in complex systems: between two successive collisions, the trajectory of the complex systems structural units is a straight line that becomes non-differentiable at the impact point. Considering that all of the collision impact points form an uncountable set of points, it results that the trajectories of the complex systems' structural units become continuous, but non-differentiable curves.

Since the non-differentiability (fractality) appears as a fundamental property of the complex systems dynamics, it seems necessary to construct a corresponding non-differentiable complex systems physics. We assume that the complexity of interactions in the complex system dynamics is replaced by non-differentiability (fractality). This topic (fractal motion) was systematically developed using either the scale relativity theory $[6,7]$ or the extended scale relativity theory, i.e., the scale relativity theory in an arbitrary constant fractal dimension $[8,9,11]$.

Some important consequences result $[8,9,11]$ :

(i) The fractal curves (the trajectories of the complex system structural units) are explicitly scale resolution dependent, i.e., their lengths tend to infinity when the scale resolution tends to zero.

(ii) The complex system dynamics is described through fractal variables, i.e., mathematical functions depending on both the space-time coordinates and the scale resolution, since the differential time reflection invariance is broken.

(iii) The differential of the spatial coordinate field is expressed as a sum of two differentials, one of them being scale resolution independent (differential part) and the other one being scale resolution dependent (fractal part).

(iv) The non-differentiable part of the spatial coordinate satisfies a fractal equation.

(v) The differential time reflection invariance is recovered by means of a complex operator (non-differentiable operator). In particular, by applying this operator to the spatial coordinate field, it results a complex velocity field, the real part being differentiable and scale resolution independent, and the imaginary one is fractal and scale resolution dependent.

(vi) The explanation of the complex operator by means of a generalized statistical fluid-like description (fractal fluid) and its implementation as a covariant derivative (with the status of motion operator in the analysis of complex system dynamics) are given.

The following results are obtained [8,9,11,12]:

(a) The acceptance of a scale covariance principle implies, by applying the covariant derivative to the complex velocity field, the equations of the structural unit geodesics of the complex system.

(b) For irrotational motions of the fractal fluid, a case in which the complex velocity field is generated by a complex scalar field, the geodesics equation reduces to a fractal Schrödinger-type equation. Then, the deterministic trajectories are replaced by a "collection of potential routes", so that both the concept of "definite position" is replaced by that of an ensemble of positions having a definite probability density, and the concept of "particle" (structural units of the complex system) is substituted with the geodesics of the Schrödinger-type themselves.

In such a frame, concepts, such as fractal entropy, fractal information, informational fractal entropy, etc., and some implications of these concepts are given [11,12].

In this paper, the role of the fractal information in the generation of constitutive laws of material, of its own differential geometry, of a variational principle, etc., at any resolution scale are analyzed in the extended scale relativity theory framework $[8,9,11,12]$. 


\section{Constitutive Laws of Material by Means of Fractal Information}

Let us consider, for now, from an abstract position of view, a complex system that is described by a probability density as a function of position $r$, time $t$ and scale resolution $d t, \rho(r, t, d t)$, normal to a unit as required:

$$
\int_{\mathcal{D}} \rho(r, t, d t) d r=1
$$

where $\mathcal{D}$ is the spatial expansion domain of the considered complex system.

The extended scale relativity theory formalism states that there is usually a complex wave function, $\Psi(\boldsymbol{r}, t, d t)$, which describes the complex system dynamics. Specifically, the function $\Psi$ is a solution of the Schrödinger-type equation (see Appendixes A, B and C, in particular from Appendix $\mathrm{C}$ and $[8,9,11])$ :

$$
-2 m A^{2} \Delta \Psi+V(r, t) \Psi=2 i m A \frac{\partial \Psi}{\partial t}, A=\lambda(d t)^{\left(2 / D_{F}\right)-1}
$$

where $V(r, t)$ is a potential of the forces that act on the complex system, $m$ is the rest mass of the "structural unit", $\lambda$ is a coefficient associated with the fractal-non-fractal transition and $D_{F}$ is the fractal dimension of the motion curve. For $D_{F}$, any definition can be accepted (Kolmogorov fractal dimension, Hausdorff-Besikovici fractal dimension [10,13], etc.), but once such a definition is accepted for $D_{F}$, it has to be constant over the entire dynamics analysis. Let us note that both Equation (2) and the potential are largely working assumptions that result, on the one hand, from the normal development of the dynamic systems [6,7] description and, on the other, from comparing the results obtained through them with the experiment.

The wave function $\Psi$ is related to the probability density $\rho$ through:

$$
\rho(r, t, d t)=|\Psi(\boldsymbol{r}, t, d t)|^{2}
$$

We note that in [11], we have defined through Equation (3) both the fractal entropy concept in the form:

$$
S_{Q}=\ln \rho(r, t, d t)
$$

and also that of fractal information:

$$
I_{Q}=-\ln \rho(r, t, d t)
$$

We ask the following: taking into consideration the probability density $\rho(r, t, d t)$ and the fractal setup given by Equations (2) and (3), what conditions must $\rho(\boldsymbol{r}, t, d t)$ meet in order to satisfy the latter?

In order to define this conditions, let us observe that from Equation (3), the most general form of the function can be deduced, in such a way that Equation (3) is always satisfied:

$$
\Psi(\boldsymbol{r}, t, d t)=R(\boldsymbol{r}, t, d t) \cdot e^{i S(r, t)}
$$

where $R=\sqrt{\rho}$ is an amplitude, admitting for the square root the arithmetical (positive) determination and $S$ is a phase. Starting from Equations (2) and (4), differential relations and easily recognizable quantities from a hydrodynamical position of view will be highlighted, their defining being the necessary condition for the viability of the setup given by Equations (1)-(4).

To start, let us replace Equation (4) in the Schrödinger-type Equation (2). A complex equation with partial derivatives will be obtained. Its advantage is just this: it can be split into a two equations with partial derivatives system that has only real quantities. The mathematical approach largely follows [7].

By replacing Equation (4) in Equation (2), we obtain the following:

$$
\frac{\partial R}{\partial t}=-A\left(R \nabla^{2} S+2 \nabla R \cdot \nabla S\right) ; \quad-2 m A R \frac{\partial S}{\partial t}=V R-2 m A^{2}\left[\nabla^{2} R-R(\nabla S)^{2}\right]
$$


The first equation from Equation (5) offers an interpretation for the time evolution of the function $\rho(r, t, d t)$ : taking into consideration that $R=\sqrt{\rho}$, it becomes:

$$
\frac{1}{\sqrt{\rho}} \frac{\partial \rho}{\partial t}=-2 A\left(\sqrt{\rho} \nabla^{2} S+\frac{1}{\sqrt{\rho}} \nabla \rho \nabla S\right)
$$

Now, we must observe the following, in order to legitimize the upcoming calculus, Equations (3) and (4) clearly show that, when $\Psi$ is null, $\rho$ is also null, and vice versa. Therefore, when $\rho$ is null and $S$ must be null, the gradients from the right side of the equation are not present. This would be a trivial case, but it shows that the probability density $\rho$ is inherently related to the microscopic phenomena described by the Schrödinger-type equation. Therefore, in order for the above written equations to make sense, it is sufficient to admit that $\rho \neq 0$. In this context, the multiplication by $\sqrt{\rho}$ of the right side of Equation (6) is legitimate. Following this, we can observe the divergence of the vector $\rho \nabla S$. We obtain the continuity equation:

$$
\frac{\partial \rho}{\partial t}+2 A \nabla(\rho \nabla S)=0
$$

for a "fluid" of density $\rho$ that flows by a velocity field specified by the time and position vectorial function:

$$
v=2 A \nabla S
$$

This is the so-called "probability fluid", and Equation (7) provides the probabilistic interpretation of extended scale relativity $[8,9,11,12]$ : it shows that the time variation of the probability density is the source of the vectorial field $\rho v$. In relation to the wave function $\Psi, \rho v$ has the known expression:

$$
\rho v=i A\left(\Psi \nabla \Psi^{*}-\Psi^{*} \nabla \Psi\right)
$$

and Equation (7) makes certain the fact that the unitary normalization of the wave function Equation (1), once chosen, is kept indefinite of time if it evolves according to the Schrödinger-type equation.

These equations do not bring forward anything new about the probabilistic interpretation of extended scale relativity. However, they show that all of the further deductions will not contradict the usual scale relativity setup [6,7]. The novelty appears when the second equation of Equation (5) is explicated. It can be written that:

$$
-2 m A \frac{\partial S}{\partial t}=V+2 m A^{2}(\nabla S)^{2}-2 m A^{2} \frac{\nabla^{2} \sqrt{\rho}}{\sqrt{\rho}}
$$

or, by separating terms,

$$
2 m A \frac{\partial S}{\partial t}+2 m A^{2}(\nabla S)^{2}=-\left(V-2 m A^{2} \frac{\nabla^{2} \sqrt{\rho}}{\sqrt{\rho}}\right)
$$

The fact that the potential of the forces acting in the system is "altered" by the following term can be observed:

$$
Q(r, t, d t)=-2 m A^{2} \frac{\nabla^{2} \sqrt{\rho}}{\sqrt{\rho}}
$$

which, as such, must itself play the role of a potential. Because $Q$ derives from the probability density as a position function, it means that its source must be the spatial variation of the probability density. The emergence of this term, which accounts for the local spatial irregularities of the probability density, is something entirely new related to the usual extended scale relativity setup, and it requires an adequate interpretation. 
By applying the operator $\nabla$ (grad) to Equation (10), taking into account Equation (8) and by multiplying after by $\rho$, we obtain:

$$
\rho \frac{\partial(m v)}{\partial t}=\frac{\partial(m \rho v)}{\partial t}-m v \frac{\partial \rho}{\partial t}
$$

and:

$$
\rho v \nabla(m \boldsymbol{v})=\nabla(m \rho \boldsymbol{v} \cdot \boldsymbol{v})-m \boldsymbol{v} \nabla(\rho \boldsymbol{v})
$$

where we wrote $v \cdot v$ instead of $v^{2}$ in order to highlight the fact that this is not the simple scalar product, but the dyadic one, i.e., the tensor composed of $v_{\mu} v_{v}$. By replacing these in Equation (12) and taking into account Equations (7) and (8), we will finally obtain:

$$
\frac{\partial(m \rho v)}{\partial t}+\nabla(m \rho v \cdot v)=-\rho \nabla\left(V-2 m A^{2} \frac{\nabla^{2} \sqrt{\rho}}{\sqrt{\rho}}\right)=-\rho \nabla(V+Q)
$$

Equation (13) is an inhomogeneous continuity equation, its inhomogeneity being given by the right side of the equation. It refers to the impulse current, and it is known in the field of hydrodynamics as the Navier-Stokes equation (for details, see [14]). In its usual interpretation, Equation (13) shows that the time variation of the momentum density is the source of the "kinetic energy" current density, i.e., the $m \rho v \cdot v$ tensor, to which one more term is being added, the one that accounts for the force density $\rho \nabla V$ and for a "hidden", for now, force density $\rho \nabla Q$, that is to be interpreted later on.

Normally, $\rho \nabla V$ must be responsible for those forces that derive from a "central" potential of the system. As we mentioned before, the central potential is largely an assumption, and in many physics problems, if not in the majority of them, it is necessary to admit a "non-centrality" (non-localization) of the potential. It is therefore easy to understand that we will describe the "non-centrality" to the term $Q$ from Equation (13). Thus, the force $\rho \nabla Q$ must be responsible for the local "non-centrality" caused by the random fluctuations of the probability density. Indeed, we will show that the term $Q$ is more likely due to some velocities, deriving from a "kinetic" energy and not from a potential energy. In order to do this, let us notice that Equation (11) can be written:

$$
Q=-2 m A^{2}\left[\frac{\nabla^{2} \rho}{\rho}-\frac{1}{2}\left(\frac{\nabla \rho}{\rho}\right)\right]
$$

Furthermore, taking into account that:

$$
\nabla\left(\frac{\nabla \rho}{\rho}\right)=\frac{\nabla^{2} \rho}{\rho}-\left(\frac{\nabla \rho}{\rho}\right)^{2}
$$

the expression from the right parenthesis of relation Equation (14) becomes:

$$
\nabla \nabla \ln \rho+\frac{1}{2}(\nabla \ln \rho)^{2}
$$

and Equation (14) itself becomes:

$$
Q=-2 m A^{2}\left[\nabla \nabla \ln \rho+\frac{1}{2}(\nabla \ln \rho)^{2}\right]
$$

Let us note that through Equation (15), both the fractal entropy and the fractal information make explicit their functionality.

The vectorial meaning of the differential calculus from Equation (15), as well as from earlier relations, is to be understood from right to left in each monom, starting from the fact that $\rho$ and $\ln \rho$ are scalar functions of position and time. For example, $\nabla \ln \rho,(\operatorname{grad}(\ln \rho))$, is a vector, while $\nabla \nabla \ln \rho$ is a scalar $(\Delta \ln \rho)$, (div. grad $(\ln \rho))$. Therefore, by applying the $\nabla$ (grad) operator to $Q$ from Equation (15), 
a vector will result, which, due to the derivatives transposition, will finally be not the gradient of a scalar, but the divergence of a tensor.

Indeed, by applying the $\nabla$ (grad) operator to $Q$ from Equation (15), it results:

$$
\begin{aligned}
\nabla Q & =-2 m A^{2}\left[\nabla \nabla \nabla \ln \rho+\frac{\nabla \rho}{\rho} \nabla \nabla \ln \rho\right]= \\
& =-2 m \rho^{-1} A^{2}[\rho \nabla \nabla \nabla \ln \rho+\nabla \rho \nabla \nabla \ln \rho]= \\
& =-2 m \rho^{-1} A^{2} \nabla(\rho \nabla \nabla \ln \rho)
\end{aligned}
$$

or:

$$
\rho \nabla Q=-2 m A^{2} \nabla \cdot(\rho \nabla \nabla \ln \rho)
$$

This time, in Equation (17), the tensor (dyadic) appears:

$$
\nabla_{\mu} \nabla_{v} \ln \rho
$$

so that the right side of Equation (17) must be the divergence of a tensor. This tensor, which can be written as:

$$
\tau_{\mu v}=2 m A^{2} \rho \nabla_{\mu} \nabla_{v} \ln \rho=2 m A^{2}\left(\nabla_{\mu} \nabla_{v} \ln \rho-\frac{\nabla_{\mu} \rho \cdot \nabla_{\nu} \rho}{\rho}\right)
$$

is, as it can be seen, symmetric, and Equation (17) has the form:

$$
\nabla \cdot \tau+\rho \nabla Q=0
$$

This equation can be interpreted not in the confines of hydrodynamics, but in the framework of the solid deformation theory for any scale: it represents the equilibrium equation for the "stress" tensor $\tau$, for a fractal body that possesses volumetric forces continuously distributed within the density $\rho \nabla Q$, as a function of position and time. For now, this analogy is purely a formal one. In the following, we will see that it can represent the real situation in a microstructured solid for any scale.

Let us note that Equation (19) written in the form:

$$
\tau_{\mu \nu}=2 m A^{2} \exp \left(S_{Q}\right) \nabla_{\mu} \nabla_{\nu} S_{Q}=-2 m A^{2} \exp \left(-I_{Q}\right) \nabla_{\mu} \nabla_{\nu} I_{Q}
$$

highlights the important role of the fractal entropy, but also that of the fractal information in defining the "stress" tensor.

Taking into account Equation (20), we note that the impulse conservation Equation (13) becomes:

$$
\frac{\partial(m \rho v)}{\partial t}+\nabla \Pi=-\rho \nabla V
$$

where $\Pi$ is the tensor composed by:

$$
\Pi_{\mu v}=m \rho v_{\mu} v_{v}-\tau_{\mu v}
$$

with $\tau_{\mu \nu}$ being given by Equation (19). It results that the effective momentum flux density $\Pi_{\mu \nu}$ is given by the momentum flux density caused by a particles current, i.e., $m \rho v_{\mu} v_{v}$, from which a tensor acting in the system, responsible for the volumetric forces density $\rho \nabla Q$, is extracted. Because usually it can be assumed that the energy current density is diminished due to dissipations in the system, this means that $\tau_{\mu v}$ must represent a dissipated energy density. We will show that this is indeed the case and that the tensor $\tau$ can be associated with a dynamic viscosity. If we define a velocity $u$ in the form:

$$
\boldsymbol{u}=A \nabla \ln \rho
$$


it results:

$$
\tau_{\mu v}=\eta\left(\frac{\partial u_{\mu}}{\partial x^{v}}+\frac{\partial u_{v}}{\partial x^{\mu}}\right)
$$

Taking into account the symmetry of the tensor $\tau$,

$$
\eta=\frac{1}{2} A \rho
$$

has the role of a dynamic viscosity coefficient [8,9]. These analogies offer an adequate image for the description of the fractal solid deformation. Indeed, because the solid's particles are characterized by relatively stable positions in the crystalline lattice or, in instability cases, by low movements of isolated or grouped particles, then the current velocity is null in all of the body's spatial domain positions, and in this case, Equation (21) simply becomes an equilibrium equation:

$$
\nabla \tau=\rho \nabla V
$$

which shows that, for a body in equilibrium, the attractive forces (repulsive) are balanced by the "dissipative" forces, the latter having here the role of repulsive forces if the potential $V$ is attractive, or the role of attractive forces if the potential $V$ is repulsive. The above equation could correspond to a "constitutive law of material", which is valid at any scale resolution.

In the usual formalism of extended scale relativity, we would not get this result, because in that case, only the vectorial field given by the current velocity is the base variable, its annulment equaling the annulment of the function $\Psi$ and, thus, the annulment of the probability density. In our interpretation, the annulment of the current velocity $v$ has no effect other than the fact that the wave function is a complex number with a constant phase factor, but of modulus $\sqrt{\rho}$ that varies only with position, but not with time, because Equation (7) with $v=0$ shows that $\partial \rho / \partial t=0$ or $\rho$ is constant in time.

Therefore, if we want to describe the microscopic behavior of the solid starting from its microstructure, it is necessary to begin with relations, such as Equations (23) and (24), for which we must introduce new supplementary assumptions:

(i) The $\eta$ coefficient from Equation (24) must be a tensor, which accounts for the crystalline lattice symmetry.

(ii) Then, let us observe that the moving velocity of the solid's particles is not determined as being the temporal derivative of the movement in itself, but as deriving from a potential given by the probability density. This results in the intrinsic movements in the solid being only "sub-products" of the theory, looking like $\int u d t$, but raising a question about the deformation as a symmetric gradient of these movements.

(iii) These facts bring forward the time problem. According to the previous observations, the density probability is constant in time and so is the velocity field. Experience contradicts however this deduction, which compels us to change our view of the solid. We conceive thusly the solid as a proper system of particles that are correctly defined by harmonic oscillators, spatially ordered in the crystalline lattice. This means that the harmonic oscillators vibrate around equilibrium positions, i.e., the points of the crystalline lattice. They can be considered identical with respect to vibrational properties, the difference being made only by their position in the crystalline lattice. These oscillators' ensemble can be described by a probability function $\rho$, which is constant in time, because the oscillators do not move from their positions. The "time constant" is too an exact term for what we intend to present: due to chaotically-distributed instant spacial states around the equilibrium positions and due to external constraints, of course related to the position they occupy, the oscillators might accidentally interact, low movements and thus repositioning occurring. This repositioning comes as an accommodation of the oscillators to the given conditions or, better put, an accommodation of the crystalline lattice to the internal and external constraints. This information must be present in an equation like Equation (25). 
Thus, the probability density itself varies in time, but it does so slowly, that it is practically insignificant, or in other words, it significantly varies only on another time scale. The cause of this variation is therefore the ensemble, singular oscillator coupling, or else put the "oscillator, lattice coupling", a complex phenomenon that relates the local disordered and ordered movements to the ensemble.

The idea of the existence of a time scale determined by the deformable solid intrinsic phenomena is not a novelty [15]. It is also present in statistical thermodynamics [15-17], due to the necessity of correlating the irreversible phenomena to the second law of thermodynamics, which stipulates the time growth of entropy. Only in our opinion, this idea does not reflect a practical reality if we only limit ourselves to describing the deformable body as a continuum, description in which geometry, mechanics and thermodynamics come together only at the exterior and, therefore, in a totally artificial way.

As a consequence, the fractal entropy and the fractal information, respectively, prove to be fundamental in establishing constitutive laws of material, which are valid at any scale resolution. Moreover, the problem of time and that of its relationship with thermodynamics can be thus discussed.

\section{Differential Geometry by Means of Fractal Information}

The results from the previous section impose two velocities fields: the standard velocity Equation (8), which is differentiable and scale resolution independent, and the fractal velocity Equation (23), which is non-differentiable and scale resolution dependent. Let us note that the fractal velocity can be directly related through Equation (23) with the fractal entropy or the fractal information, respectively.

Since the two real velocities simultaneously act, we can introduce through the relation (for details, see [11]):

$$
\hat{V}=-2 i A \nabla \ln \Psi(r, t, d t)
$$

the complex velocities field:

$$
\hat{v}=v-i u, \quad v=2 A \nabla S, \quad u=A \nabla \ln \rho
$$

generated by the complex scalar potential:

$$
\Phi=-2 i A[\ln R(r, t, d t)+i S(r, t)]
$$

From here, by means of the differential one-forms:

$$
\bar{\omega}^{1}=d \Phi=2 A\left(d S+i \frac{d R}{R}\right), \quad \bar{\omega}^{2}=d \bar{\Phi}=2 A\left(d S-i \frac{d R}{R}\right)
$$

we can introduce the differential two-form:

$$
d s^{2}=\bar{\omega}^{1} \bar{\omega}^{2}=d \Phi d \bar{\Phi}=4 A^{2}\left[d S^{2}+\left(\frac{d R}{R}\right)^{2}\right]
$$

For the condition:

$$
d S=d \varphi=\frac{d u}{v}
$$

and with the notations:

$$
h=u+i v, \quad \bar{h}=u-i v, \quad R \equiv v, k_{0}^{2}=4 A^{2}
$$

the differential two-form, Equation (31), represents the metric of the Lobachevsky plane in the Poincare representation $[15,18-21]$ :

$$
\frac{d s^{2}}{k_{0}^{2}}=\frac{d h d \bar{h}}{(h-\bar{h})^{2}}=\frac{d u^{2}+d v^{2}}{v^{2}}
$$

We shall come back later on the condition, Equation (32). 
The metric, Equation (34), is invariant with respect to the group of homographies, a continuous group with three essential parameters [15,21].

Consider, therefore, a real homographic transformation on the variable $h$ :

$$
h^{\prime}=\frac{a h+b}{c h+d}, a, b, c, d \in \mathbb{R}, a d-b c \neq 0
$$

The conjugates of the two complex quantities $h$ and $h^{\prime}$ are connected, obviously, by the same transformation:

$$
\overline{h^{\prime}}=\frac{a \bar{h}+b}{c \bar{h}+d}
$$

Transformation (35) and (36) form a group of two variables with three parameters (one of the quantities $a, b, c, d$ is not essential), as can easily be verified. This group leaves invariant the differential quadratic form:

$$
-4 \frac{d h d \bar{h}}{(h-\bar{h})^{2}}
$$

Indeed, in view of transformation (35) and (36), we have:

$$
d h^{\prime}=\frac{(a d-b c) d h}{(c h+d)^{2}}, \quad d \overline{h^{\prime}}=\frac{(a d-b c) d \bar{h}}{(c \bar{h}+d)^{2}}, \quad h^{\prime}-\overline{h^{\prime}}=\frac{(a d-b c)(h-\bar{h})}{(c h+d)(c \bar{h}+d)}
$$

which immediately yields:

$$
\frac{d h^{\prime} d \overline{h^{\prime}}}{\left(h^{\prime}-\overline{h^{\prime}}\right)^{2}}=\frac{d h d \bar{h}}{(h-\bar{h})^{2}}
$$

The metric of the Lobachevsky plane can be produced as a Calylean metric of an Euclidean plane, for which the absoluteness is a circle with unit radius [22]. This way, the Lobachevsky plane can be put into biunivocal correspondence with the interior side of this circle. The general procedure of metrization of a Calylean space starts with the definition of the metric as an anharmonic ratio, being extensively described in many classical volumes (see, for example, [23-25]), so that we shall skip the details and give only the final results, which are necessary in our following investigation. Let us suppose that the absoluteness of the space is represented by the quadratic form $\Omega(X, Y)$, where $X$ denotes any vector. The Calylean metric is then given by the differential quadratic form:

$$
\frac{d s^{2}}{k_{0}^{2}}=\frac{\Omega(d X, d X)}{\Omega(X, X)}-\frac{\Omega^{2}(d X, d X)}{\Omega^{2}(X, X)}
$$

where $\Omega(X, Y)$ is the duplication of $\Omega(X, X)$ and $k_{0}$ a constant connected to the space curvature.

In case of the Lobachevsky plane, we have:

$$
\Omega(X, X)=1-x^{2}-y^{2}, \quad \Omega(X, d X)=-x d x-y d y, \quad \Omega(d X, d X)=-d x^{2}-d y^{2}
$$

which yields:

$$
\frac{d s^{2}}{k_{0}^{2}}=\frac{\left(1-y^{2}\right) d x^{2}+2 x y d x d y+\left(1-x^{2}\right) d y^{2}}{\left(1-x^{2}-y^{2}\right)^{2}}
$$

Performing now the coordinate transformation:

$$
x=\frac{h \bar{h}-1}{h \bar{h}+1}, \quad y=\frac{h+\bar{h}}{h \bar{h}+1}
$$


the metric Equation (40) becomes identical to Equation (34):

$$
\frac{d s^{2}}{k_{0}^{2}}=-4 \frac{d h d \bar{h}}{(h-\bar{h})^{2}}
$$

As is easily seen, the absoluteness $1-x^{2}-y^{2}=0$ goes to the straight $\operatorname{line} \operatorname{Im} h=0$, while straight lines of the Euclidean plane go to circles with centers on the real axis of the complex plane $(h)$.

The previous theory is applicable to any case of the existence of a real conic, being easy to demonstrate that the Calylean metric attached to this conic is the metric generated by the transformation group, which leaves it invariant.

As we have mentioned, the group Equation (35) is a group in two variables with three parameters. Writing explicitly its transformations, so that the identical transformation corresponds to the null values of parameters, we can conveniently write them as:

$$
h^{\prime}=\frac{h+a^{1}}{a^{3} h+a^{2}+1}
$$

where $a^{1}, a^{2}, a^{3}$ are the real group parameters. Next, let us express its infinitesimal transformation [21], i.e., those transformations characterized by infinitesimal values of parameters, and expand Equation (43) together with its complex conjugate about the parameters origin $\left(a^{1}=a^{2}=a^{3}=0\right)$. The result is:

$$
h^{\prime}=h+a^{1}-h a^{2}-h^{2} a^{3}, \quad \overline{h^{\prime}}=\bar{h}+a^{1}-\bar{h} a^{2}-\overline{h^{2}} a^{3}
$$

where, this time, $a^{1}, a^{2}, a^{3}$ are infinitely small quantities of the first order, while their higher powers have been neglected.

According to Equation (44), one and the same transformation $h \rightarrow h^{\prime}$ corresponds to an infinite number of values of parameters $a^{1}, a^{2}, a^{3}$. This result can be easily verified: solving the system Equation (44) with respect to $a^{1}$, we realize that it is compatible undetermined. It is said that the group is multiply transitive; there is an infinity of its homographies, which makes its possible transition $h \rightarrow h^{\prime}$.

Recalling the significance of $h$ variables as being field potentials from the physical point of view would be desirable for the group to be simply transitive: to a given system of parameters $a^{1}, a^{2}, a^{3}$ corresponds one transition $\left(h \rightarrow h^{\prime}\right)$, and only one. This desire is purely classical, and corresponds, for example, to the construction of Maxwell's equations, under given initial conditions.

A necessary condition for a group to be simply transitive is, obviously, that the number of variables equals the number of parameters. In this case, Equation (44) should contain three equations, instead of two. If, in addition, the matrix of coefficients of $a^{1}, a^{2}, a^{3}$ would be of rank three, then certainly the group would be simply transitive.

Keeping in mind the origin of this group, the only possibility is to add a new field variable (whose significance shall be explained later on). This operation can be done in many ways, under condition of keeping the structure of the starting group. As one can observe by examining Equation (44), this condition is given by the infinitesimal operators:

$$
\hat{B}_{1}=\frac{\partial}{\partial h}+\frac{\partial}{\partial \bar{h}^{\prime}}, \quad \hat{B}_{2}=-h \frac{\partial}{\partial h}-\bar{h} \frac{\partial}{\partial \bar{h}^{\prime}}, \quad \hat{B}_{3}=-h^{2} \frac{\partial}{\partial h}-\bar{h}^{2} \frac{\partial}{\partial \bar{h}}
$$

through the equations:

$$
\left[\hat{B}_{1}, \quad \hat{B}_{2}\right]=-\hat{B}_{1}, \quad\left[\hat{B}_{2}, \hat{B}_{3}\right]=-\hat{B}_{3},\left[\hat{B}_{3}, \hat{B}_{1}\right]=-2 \hat{B}_{2}
$$

Rewriting the infinitesimal transformation Equations (44)-(46) in three variables as:

$$
h^{\prime}=h+a^{1}-h a^{2}-h^{2} a^{3}, \quad \overline{h^{\prime}}=\bar{h}+a^{1}-\bar{h} a^{2}-\overline{h^{2}} a^{3}, \quad k^{\prime}=k+\gamma(h, \bar{h}, k) a^{3}
$$


where $k$ is the new variable introduced due to already mentioned reasons; the first two differential operators of Equation (45) do not change, while the third becomes:

$$
\hat{L}_{3}=\hat{B}_{3}+\gamma \frac{\partial}{\partial k}
$$

Imposing on the new operators the same structural relations as those given in Equation (46), it follows that $\gamma$ is defined up to a multiplicative function of the new variable:

$$
\gamma(h, \bar{h}, k)=\psi(k)(h-\bar{h})
$$

Choosing, for reasons that soon shall become obvious, $\psi(k)=-k$, it follows that the infinitesimal operators of the new simply transitive group (it can be easily verified that the system Equation (47) has a unique solution in $a^{1}, a^{2}, a^{3}$ under condition $h-\bar{h} \neq 0, k \neq 0$ ) are given by:

$$
\hat{L}_{1} \equiv \hat{B}_{1}, \quad \hat{L}_{2} \equiv \hat{B}_{2}, \quad \hat{L}_{3} \equiv-\hat{B}_{3}-(h-\bar{h}) k \frac{\partial}{\partial k}
$$

As can be observed, $k$ has to be a unimodular complex variable. This can be proven by solving the equation:

$$
X_{3} f=0
$$

It is satisfied if and only if:

$$
k=\operatorname{constant} \frac{\bar{h}}{h}
$$

If the constant is adequately adjusted, we can limit ourselves, indeed, to considering $k$ as a unimodular factor, but without being the ratio between $h$ and $\bar{h}$. This was the reason for choosing $\psi=-k$ in Equation (49): if we set $k=e^{i \varphi \prime}$, then we shall have $k \frac{\partial}{\partial k}=\frac{\partial}{\partial \varphi^{\prime}}$, which means the derivative with respect to the phase of $k$.

As can easily be verified, the infinitesimal relations Equation (49) correspond to the finite transformations:

$$
h^{\prime}=\frac{h+a^{1}}{a^{3} h+a^{2}+1}, \quad k^{\prime}=\frac{a^{3} \bar{h}+a^{2}+1}{a^{3} h+a^{2}+1} k
$$

and this is the Barbilian group, named after Romanian mathematician Dan Barbilian [23-25]. Here are some properties of this group.

Its structure is given by Equation (49), and consequently, the structure constants are:

$$
C_{12}^{1}=C_{23}^{3}=-1, \quad C_{31}^{2}=-2
$$

the rest of them being zero. Therefore, the invariant quadratic form is given by the quadratic tensor of the group [26], that is:

$$
C_{\alpha \beta}=C_{\alpha \nu}^{\mu} C_{\beta \mu}^{v}
$$

where summation over repeated indices is understood. Using Equations (52) and (53), the tensor $C_{\alpha \beta}$ writes:

$$
C_{\alpha \beta}=\left(\begin{array}{lrr}
0 & 0 & -4 \\
0 & 2 & 0 \\
-4 & 0 & 0
\end{array}\right)
$$

meaning that the invariant metric of the group has the form:

$$
\frac{d s^{2}}{k_{0}^{2}}=\omega_{0}^{2}-4 \omega_{1} \omega_{2}
$$


where $k_{0}$ is an arbitrary factor and $\left(\omega_{\alpha}\right)$ three differential one-forms, absolutely invariant through the group. These one-forms have the form:

$$
\omega_{0}=i\left(\frac{d k}{k}-\frac{d h+d \bar{h}}{h-\bar{h}}\right), \quad \omega_{1}=\omega_{2}=\frac{d h}{k(h-\bar{h})}
$$

and the metric becomes:

$$
\frac{d s^{2}}{k_{0}^{2}}=-\left(\frac{d k}{k}-\frac{d h+d \bar{h}}{h-\bar{h}}\right)^{2}+4 \frac{d h d \bar{h}}{k(h-\bar{h})^{2}}
$$

It is worthwhile to mention a property connected to the integral geometry: the Barbilian group Equation (51) is measurable. Indeed, it is simply transitive, and since its structure vector [26]:

$$
C_{\alpha}=C_{v \alpha}^{v}
$$

is identically null, as can be seen from Equation (52), this means that it possess an invariant function given by:

$$
F(h, \bar{h}, k)=-\frac{1}{(h-\bar{h})^{2} k}
$$

which is the inverse of the modulus of determinant of the linear system Equation (47) in unknowns $\left(a^{i}\right)$.

As a result, in the space of the field variables $(h, \bar{h}, k)$ can a priori be constructed a probabilistic theory $[15,21]$, based on the elementary probability:

$$
d P(h, \bar{h}, k)=-\frac{d h \Lambda d \bar{h} \Lambda d k}{(h-\bar{h})^{2} k}
$$

where $\Lambda$ denotes the exterior product of the one-forms.

Let us note that the complex variable $h$ (being given with the fractal information; see the substitutions Equation (33)) can become fundamental in obtaining a Jeans-type physical theory; for details, see $[15,21]$.

Finally, it is notable to observe that the Barbilian group is "latently" contained in the group of homographic transformations Equation (43). Indeed, let us focus our attention on metric Equation (57): it reduces to metric Equation (42) for $\omega_{0}=0$. By means of the usual relations:

$$
h=u+i v, k=e^{i \varphi}
$$

relation Equation (56) yields

$$
\omega_{0}=-\left(d \varphi+\frac{d u}{v}\right)
$$

and therefore, condition $\omega_{0}=0$ becomes:

$$
d \varphi=-\frac{d u}{v}
$$

which is the definition Equation (55) of the Levi-Civita parallelism angle. Therefore, $\omega_{0}=0$ represents the Levi-Civita parallelism of the Lobachevsky plane (for details, see [22]).

In this way, the fractal information becomes fundamental in the construction of the metrics of Lobachevsky's plane in the Poincaré representation, at any scale resolution.

\section{A Variational Principle by Means of Fractal Information}

Let us suppose that the field is described by the variables $\left(y^{l}\right)$ for which we have obtained the metric:

$$
h_{i l} d y^{i} d y^{l}
$$


in an ambient space of the metric:

$$
\gamma_{\alpha \beta} d x^{\alpha} d x^{\beta}
$$

In this situation, the field equations are derived from the variational principle:

$$
\delta \int L \gamma^{1 / 2} d^{3} x=0
$$

connected to the Lagrangian:

$$
L=\gamma^{\alpha \beta} h_{i l} \frac{\partial y^{i} \partial y^{l}}{\partial x^{\alpha} \partial x^{\beta}}
$$

In our case, the metric (64) is given by Equation (34), the field variables being $h$ and $\bar{h}$ or, equivalently, the real and the imaginary parts of $h$. Then, the Lagrangian Equation (67) takes the form:

$$
L=\gamma^{\alpha \beta} \frac{\frac{\partial h}{\partial x^{\alpha}} \frac{\partial \bar{h}}{(h-\bar{h})^{2}}}{(h-\bar{h})^{2}}
$$

so that through the variational principle Equation (66), the field equations become:

$$
(h-\bar{h}) \nabla^{2} h=2 \nabla h \nabla h, \quad(h-\bar{h}) \nabla^{2} \bar{h}=2 \nabla \bar{h} \nabla \bar{h}
$$

A solution of this equation has the form:

$$
\begin{gathered}
h=-i \frac{\cosh \psi-e^{-i \alpha} \sinh \psi}{\cosh \psi+e^{-i \alpha} \sinh \psi}, \quad \bar{h}=i \frac{\cosh \psi-e^{i \alpha} \sinh \psi}{\cosh \psi+e^{i \alpha} \sinh \psi} \\
\nabla^{2} \psi=0
\end{gathered}
$$

with $\alpha$ real. The Equation (71) represents an harmonic map from the usual space into the Lobachevsky plane having the metric Equation (34), provided $\psi$ is a solution of the Laplace equation in free space. Therefore, if this variational principle is accepted as a starting point, the main purpose of the field research would be the produced metrics of Lobachevsky (or related to them).

In consequence, by means of the variational principle Equation (66) of Lagrangian Equation (67) or in particular Equation (68), the fractal information becomes a fundamental component in the construction of a field theory, at any scale resolution.

\section{Conclusions}

The main conclusions of this paper are the following:

(i) Starting from a Schrödinger-type equation as geodesics of a fractal space in the frame of the extended scale relativity theory, it is proven that the velocities' field is complex. Its real part is the standard velocity, which is differentiable and scale resolution independent, while its imaginary part is the fractal velocity, which is non-differentiable and scale resolution dependent.

(ii) Through a Madelung-type choice of the wavefunction in the Schrödinger geodesic, the following two parts are separated: the real one (which implies the impulse conservation law) and the imaginary one (which implies the states density conservation law). The two conservation laws constitute the fractal hydrodynamic equations system.

(iii) The differential velocity is put in correspondence with the wavefunction phase, while the fractal velocity is put in correspondence with the wavefunction amplitude and, thus, with the states density, through the square amplitude. By such a procedure, the proportionality between the fractal velocity and the fractal information gradient is highlighted.

(iv) The fractal velocity and, thus, the fractal information gradient are responsible (through the fractal potential gradient) for the presence of a force-type term (fractal force) in the impulse conservation law. At equilibrium, this term can be put in correspondence with the divergence of the tensor of 
"stresses", and this imposes a constitutive law of material. In other words, the tensor of "stresses" becomes a measure of the fractal information "fluctuations".

(v) From the perspective of a two-form of a complex scalar potential of velocities (potential, which contains explicitly the fractal information as its imaginary part), one obtains the correspondence with the metric of the Lobachevsky plane in the Poincaré representation. It thus results in the invariance of the two-form with respect to the homographic transformations group (the Barbilian group), when one defines a parallel transport of vectors in the sense of Levi-Civita (the vector origin moves on geodesics, the angle between the vector and the tangent to the geodesic at the current point being permanently constant). The group is measurable (that is, it possesses a function, which is integrally invariant), so that in the variables space of the group, one can construct Jeans-type probabilistic physical theories based on an elementary probability (for details, see $[15,21])$. Moreover, the fact that the metric of the Lobachevsky plane can be produced as a Caylean metric of the Euclidean space (for which the absoluteness is a circle with unit radius) proves that one can produce metrics and, thus, fractal information, at any scale resolution, independently of the Einsteinian procedure.

(vi) A variational principle has been constructed. Then, if this variational principle is accepted as a starting point, the main purpose of any field research would be to produce metrics of the Lobachevsky plane, being apart from Einstein's field equations. The field equations obtained through this variational principle represent a harmonic map from the usual space into the Lobachevsky plane having the metric in Poincaré's representation. In other words, through this variational principle, one can produce fractal informational that is explicit at any scale resolution. In this sense, if the field variables define a gravitational field, then the variational principle is reducible to a Matzner-Misner-type one, while the field equations are reducible to those of Ernst-type (for details, see $[15,21]$ ). From such a perspective, the fractal information is generated together with the material structure, that is together with the induction of the own space associated with the material structure.

This paper could lead to some considerations both in fundamental scientific and other areas, such as business management, marketing, etc. A special role could be given to the economic implications of macroeconomic and microeconomic developments.

Acknowledgments: The authors are indebted to the unknown referees for their valuable remarks and suggestions in improving the paper.

Author Contributions: In this paper, Maricel Agop provided the original idea and constructed its framework. Alina Gavriluţ and Viorel Puiu Păun conducted the detailed calculation and were responsible for drafting and revising the whole paper. Dumitru Filipeanu, Florin Alexandru Luca, Constantin Grecea and Liliana Topliceanu devoted efforts to some valuable comments on the paper. All authors have read and approved the final manuscript.

Conflicts of Interest: The authors declare no conflict of interest.

\section{Appendix A. Implications of Non-Differentiability in the Dynamics of Complex Systems}

Assuming that the motions of the complex systems' structural units take place on continuous, but non-differentiable curves (fractal curves), the following consequences emerge:

1. Any continuous, but non-differentiable curve of the complex system structural units is explicitly scale resolution $\delta t$ dependent, i.e., its length tends to infinity when $\delta t$ tends to zero.

We mention that, mathematically speaking, a curve is non-differentiable if it satisfies the Lebesgue theorem, i.e., its length becomes infinite when the scale resolution goes to zero [13]. Consequently, in this limit, a curve is as zigzagged as one can imagine. Thus, it exhibits the property of self-similarity in every one of its points, which can be translated into a property of holography (every part reflects the whole) $[6,7,10,13]$.

2. The physics of the complex phenomena is related to the behavior of a set of functions during the zoom operation of the scale resolution $\delta t$. Then, through the substitution principle, $\delta t$ will be 
identified with $d t$, i.e., $\delta t \equiv d t$, and consequently, it will be considered as an independent variable. We reserve the notation $d t$ for the usual time as in the Hamiltonian complex system dynamics.

3. The complex system dynamics is described through fractal variables, i.e., functions depending on both the space-time coordinates and the scale resolution, since the differential time reflection invariance of any dynamical variable is broken. Then, in any point of the fractal curve, two derivatives of the variable field $Q(t, d t)$ can be defined:

$$
\begin{aligned}
& \frac{d_{+} Q(t, d t)}{d t}=\lim _{\Delta t \rightarrow 0_{+}} \frac{Q(t+\Delta t, \Delta t)-Q(t, \Delta t)}{\Delta t} \\
& \frac{d_{-} Q(t, d t)}{d t}=\lim _{\Delta t \rightarrow 0_{-}} \frac{Q(t, \Delta t)-Q(t-\Delta t, \Delta t)}{\Delta t}
\end{aligned}
$$

The "+" sign corresponds to the forward process, while the "-" sign correspond to the backwards one.

4. The differential of the spatial coordinate field $d X^{i}(t, d t)$ is expressed as the sum of the two differentials, one of them being scale resolution independent (differential part $d_{ \pm} x^{i}(t)$ ) and the other one being scale resolution dependent (fractal part $\left.d_{ \pm} \xi^{i}(t)\right)[6,7]$, i.e.,

$$
d_{ \pm} X^{i}(t, d t)=d_{ \pm} x^{i}(t)+d_{ \pm} \xi^{i}(t, d t)
$$

5. The non-differentiable part of the spatial coordinate field satisfies the fractal equation [6,7]:

$$
d_{ \pm} \xi^{i}(t, d t)=\lambda_{ \pm}^{i}(d t)^{1 / D_{F}}
$$

where $\lambda_{ \pm}^{i}$ are constant coefficients through which the fractalization type is specified and $D_{F}$ defines the fractal dimension of the fractal curve.

In our opinion, the processes in complex systems imply a dynamics on geodesics having various fractal dimensions. Precisely, for $D_{F}=2$, quantum-type processes are generated. For $D_{F}<2$, correlative-type processes are induced, while for $D_{F}>2$, non-correlative-type ones can be found (for details, see $[6-9,13])$.

6. The differential time reflection invariance of any dynamical variable is recovered by combining the derivatives $d_{+} / d t$ and $d_{-} / d t$ in the non-differentiable operator:

$$
\frac{\hat{d}}{d t}=\frac{1}{2}\left(\frac{d_{+}+d_{-}}{d t}\right)-\frac{i}{2}\left(\frac{d_{+}-d_{-}}{d t}\right)
$$

This is a natural result of the complex prolongation procedure [7]. Applying now the non-differentiable operator to the spatial coordinate field yields the complex velocity field:

$$
\hat{V}^{i}=\frac{\hat{d} X^{i}}{d t}=V_{D}^{i}-V_{F}^{i}
$$

with:

$$
\begin{aligned}
& V_{D}^{i}=\frac{1}{2}\left(v_{+}^{i}+v_{-}^{i}\right), \quad V_{F}^{i}=\frac{1}{2}\left(v_{+}^{i}-v_{-}^{i}\right) \\
& v_{+}^{i}=\frac{d_{+} x^{i}+d_{+} \xi^{i}}{d t}, \quad v_{-}^{i}=\frac{d_{-} x^{i}+d_{-} \xi^{i}}{d t}
\end{aligned}
$$

The real part $V_{D}^{i}$ is differentiable and scale resolution independent (differentiable velocity field), while the imaginary one $V_{F}^{i}$ is non-differentiable and scale resolution dependent (fractal velocity field).

7. In the absence of any external constraint, an infinite number of fractal curves (geodesics) can be found relating any pair of points, and this is true on all scales. Then, in the fractal space, 
all complex system structural units are substituted with the geodesics themselves (for details, see Appendix B), so that any external constraint is interpreted as a selection of geodesics by the measuring device. The infinity of geodesics in the bundle, their non-differentiability and the two values of the derivative imply a generalized statistical fluid-like description (fractal fluid). Then, the average values of the fractal fluid variables must be considered in the previously-mentioned sense, so the average of $d_{ \pm} X^{i}$ is:

$$
\left\langle d_{ \pm} X^{i}\right\rangle \equiv d_{ \pm} x^{i}
$$

with:

$$
\left\langle d_{ \pm} \xi^{i}\right\rangle=0
$$

8. The complex system dynamics can be described through a covariant derivative, the explicit form of which is obtained as follows. Let us consider that the non-differentiable curves are immersed in a three-dimensional space and that $X^{i}$ are the spatial coordinate field of a point on the non-differentiable curve. We also consider a variable field $Q\left(X^{i}, t\right)$ and the following Taylor expansion up to the second order:

$$
d_{ \pm} Q\left(X^{i}, t\right)=\partial_{t} Q d t+\partial_{i} Q d_{ \pm} X^{i}+\frac{1}{2} \partial_{l} \partial_{k} Q d_{ \pm} X^{l} d_{ \pm} X^{k}
$$

These relations are valid in any point of the space and more for the points $X^{i}$ on the non-differentiable curve, which we have selected in Equation (A9). From here, forward and backward values of Equation (A9) become:

$$
\left\langle d_{ \pm} Q\right\rangle=\left\langle\partial_{t} Q d t\right\rangle+\left\langle\partial_{i} Q d_{ \pm} X^{i}\right\rangle+\frac{1}{2}\left\langle\partial_{l} \partial_{k} Q d_{ \pm} X^{l} d_{ \pm} X^{k}\right\rangle
$$

We suppose that the average values of the variable field $Q$ and its derivatives coincide with themselves, and the differentials $d_{ \pm} X^{i}$ and $d t$ are independent. Therefore, the average of their products coincides with the product of averages. Consequently, Equation (A10) becomes:

$$
d_{ \pm} Q=\partial_{t} Q d t+\partial_{i} Q\left\langle d_{ \pm} X^{i}\right\rangle+\frac{1}{2} \partial_{l} \partial_{k} Q\left\langle d_{ \pm} X^{l} d_{ \pm} X^{k}\right\rangle
$$

Even the average value of $d_{ \pm} \xi^{i}$ is null for the higher order of $d_{ \pm} \xi^{i}$, the situation can still be different. Let us focus on the averages $\left\langle d_{ \pm} \xi^{l} d_{ \pm} \xi^{k}\right\rangle$. Using Equation (A3), we can write:

$$
\left\langle d_{ \pm} \xi^{l} d_{ \pm} \xi^{k}\right\rangle= \pm \lambda_{ \pm}^{l} \lambda_{ \pm}^{k}(d t)^{\left(2 / D_{F}\right)-1} d t
$$

where we accepted that the sign + corresponds to $d t>0$ and the sign - corresponds to $d t<0$.

Then, Equation (A11) takes the form:

$$
d_{ \pm} Q=\partial_{t} Q d t+\partial_{i} Q\left\langle d_{ \pm} X^{i}\right\rangle+\frac{1}{2} \partial_{l} \partial_{k} Q d_{ \pm} x^{l} d_{ \pm} x^{k} \pm \frac{1}{2} \partial_{l} \partial_{k} Q\left[\lambda_{ \pm}^{l} \lambda_{ \pm}^{k}(d t)^{\left(2 / D_{F}\right)-1} d t\right]
$$

If we divide by $d t$ and neglect the terms that contain differential factors (for details, see the method from [6-9]), we obtain:

$$
\frac{d_{ \pm} Q}{d t}=\partial_{t} Q+v_{ \pm}^{i} \partial_{i} Q \pm \frac{1}{2} \lambda_{ \pm}^{l} \lambda_{ \pm}^{k}(d t)^{\left(2 / D_{F}\right)-1} \partial_{l} \partial_{k} Q
$$


These relations also allow us to define the operators:

$$
\frac{d_{ \pm}}{d t}=\partial_{t}+v_{ \pm}^{i} \partial_{i} \pm \frac{1}{2} \lambda_{ \pm}^{l} \lambda_{ \pm}^{k}(d t)^{\left(2 / D_{F}\right)-1} \partial_{l} \partial_{k}
$$

Under these circumstances, taking into account Equations (A4), (A5) and (A15), let us calculate $\hat{d} / d t$. It results:

$$
\frac{\hat{d} Q}{d t}=\partial_{t} Q+\hat{V}^{i} \partial_{i} Q+\frac{1}{4}(d t)^{\left(2 / D_{F}\right)-1} D^{l k} \partial_{l} \partial_{k} Q
$$

where:

$$
\begin{aligned}
& D^{l k}=d^{l k}-i \bar{d}^{l k} \\
& d^{l k}=\lambda_{+}^{l} \lambda_{+}^{k}-\lambda_{-}^{l} \lambda_{-}^{k}, \quad \bar{d}^{l k}=\lambda_{+}^{l} \lambda_{+}^{k}+\lambda_{-}^{l} \lambda_{-}^{k}
\end{aligned}
$$

The relation Equation (A16) also allows us to define the covariant derivative:

$$
\frac{\hat{d}}{d t}=\partial_{t}+\hat{V}^{i} \partial_{i}+\frac{1}{4}(d t)^{\left(2 / D_{F}\right)-1} D^{l k} \partial_{l} \partial_{k}
$$

\section{Appendix B. Geodesics of the Complex System Structural Units}

Let us now consider the principle of scale covariance (the physics laws of the complex systems dynamics are invariant with respect to scale transformations) and postulate that the passage from the classical complex system physics of the complex systems to the non-differentiable (fractal) physics of the complex systems can be implemented by replacing the standard time derivative $d / d t$ by the non-differentiable operator $\hat{d} / d t$. Thus, this operator plays the role of the covariant derivative, namely it is used to write the fundamental equations of complex system dynamics in the same form as in the classic (differentiable) case. Under these conditions, applying the operator Equation (A18) to the complex velocity field Equation (A5), in the absence of any external constraint, the geodesics of the complex system structural units have the following form:

$$
\frac{\hat{d} \hat{V}^{i}}{d t}=\partial_{t} \hat{V}^{i}+\hat{V}^{l} \partial_{l} \hat{V}^{i}+\frac{1}{4}(d t)^{\left(2 / D_{F}\right)-1} D^{l k} \partial_{l} \partial_{k} \hat{V}^{i}=0
$$

This means that the local acceleration $\partial_{t} \hat{V}^{i}$, the convection $\hat{V}^{l} \partial_{l} \hat{V}^{i}$ and the dissipation $D^{l k} \partial_{l} \partial_{k} \hat{V}^{i}$ make their balance in any point of the non-differentiable curve. Moreover, the presence of the complex coefficient of viscosity-type $\frac{1}{4}(d t)^{\left(2 / D_{F}\right)-1} D^{l k}$ specifies that the complex system can be assimilated with a rheological medium, so it has memory, as a datum, by his own structure.

If the fractalization is achieved by Markov-type stochastic processes, which involve Lévy-type movements of the complex system structural units $[6,7,13]$, then:

$$
\lambda_{+}^{i} \lambda_{+}^{l}=\lambda_{-}^{i} \lambda_{-}^{l}=2 \lambda \delta^{i l}
$$

where $\delta^{i l}$ is the Kronecker's symbol, with the property:

$$
\delta^{i l}= \begin{cases}1, & i=l \\ 0, & i \neq l\end{cases}
$$

Under these conditions, the equation of geodesics takes the simple form:

$$
\frac{\hat{d} \hat{V}^{i}}{d t}=\partial_{t} \hat{V}^{i}+\hat{V}^{l} \partial_{l} \hat{V}^{i}-i \lambda(d t)^{\left(2 / D_{F}\right)-1} \partial^{l} \partial_{l} \hat{V}^{i}=0
$$


or more, by separating the motions on differential and fractal scale resolutions,

$$
\begin{aligned}
& \frac{\hat{d} \hat{V}_{D}^{i}}{d t}=\partial_{t} V_{D}^{i}+V_{D}^{l} \partial_{l} V_{D}^{i}-\left[V_{F}^{l}-\lambda(d t)^{\left(2 / D_{F}\right)-1} \partial^{l}\right] \partial_{l} V_{F}^{i}=0 \\
& \frac{\hat{d} \hat{V}_{F}^{i}}{d t}=\partial_{t} V_{F}^{i}+V_{D}^{l} \partial_{l} V_{F}^{i}-\left[V_{F}^{l}-\lambda(d t)^{\left(2 / D_{F}\right)-1} \partial^{l}\right] \partial_{l} V_{D}^{i}=0
\end{aligned}
$$

\section{Appendix C. Geodesics of the Complex Systems Dynamics in the Schrödinger-Type Representation}

For irrotational motions:

$$
\varepsilon_{i k l} \partial^{k} \hat{V}^{l}=0
$$

where $\varepsilon_{i k l}$ is the Levi-Civita pseudo-tensor. Then, we choose $\hat{V}^{i}$ in the form [6-9]:

$$
\hat{V}^{i}=-2 i \lambda(d t)^{\left(2 / D_{F}\right)-1} \partial^{i} \ln \psi
$$

where, for the moment, $\ln \psi$ defines the scalar potential of the complex velocity field. Substituting Equation (C2) in Equation (B3), we obtain:

$$
\begin{aligned}
\frac{\hat{d} \hat{V}^{i}}{d t} & =-2 i \lambda(d t)^{\left(2 / D_{F}\right)-1}\left\{\partial_{t} \partial^{i} \ln \psi-i\left[2 \lambda(d t)^{\left(2 / D_{F}\right)-1}\right.\right. \\
& \left.\left.\times\left(\partial^{l} \ln \psi \partial_{l}\right) \partial^{i} \ln \psi+\lambda(d t)^{\left(2 / D_{F}\right)-1} \partial^{l} \partial_{l} \partial^{i} \ln \psi\right]\right\}=0
\end{aligned}
$$

Using the identities:

$$
\begin{aligned}
& \partial^{l} \partial_{l} \ln \psi+\partial_{i} \ln \psi \partial^{i} \ln \psi=\frac{\partial_{l} \partial^{l} \psi}{\psi} \\
& \partial^{i}\left(\frac{\partial^{l} \partial_{l} \psi}{\psi}\right)=2\left(\partial^{l} \ln \psi \partial_{l}\right) \partial^{i} \ln \psi+\partial^{l} \partial_{l} \partial^{i} \ln \psi
\end{aligned}
$$

the Equation (C3) becomes:

$$
\begin{aligned}
\frac{\hat{d} \hat{V}^{i}}{d t} & =-2 i \lambda(d t)^{\left(2 / D_{F}\right)-1} \partial^{i} \times \\
& \times\left[\partial_{t} \ln \psi-2 i \lambda(d t)^{\left(2 / D_{F}\right)-1} \frac{\partial^{l} \partial_{l} \ln \psi}{\psi}\right]=0
\end{aligned}
$$

This equation can be integrated up to an arbitrary phase factor, which may be set to zero by a suitable choice of phase of $\psi$, and this yields:

$$
\lambda^{2}(d t)^{\left(4 / D_{F}\right)-2} \partial^{l} \partial_{l} \psi+i \lambda(d t)^{\left(2 / D_{F}\right)-1} \partial_{t} \psi=0
$$

For motions on Peano-type curves, $D_{F}=2[6,7,13]$, at Compton scale, $\lambda=\hbar / 2 m_{0}$, with $\hbar$, the reduced Planck's constant and $m_{0}$ the rest mass of the structural units, the relation Equation (C6) becomes the usual Schrödinger equation. The relation Equation (C6) is a Schrödinger-type equation (geodesics in the Schrödinger representation). We note that the presence of an external scalar potential $V$ modifies the Equation (C6) in the form:

$$
\lambda^{2}(d t)^{\left(4 / D_{F}\right)-2} \partial^{l} \partial_{l} \psi+i \lambda(d t)^{\left(2 / D_{F}\right)-1} \partial_{t} \psi-\frac{V}{2} \psi=0
$$


The relation Equation (C7) corresponds to the equation of motion for the "one body problem" in the Schrödinger-type representation of the non-differentiable model. The standard equation of motion for the "one body problem" in the scale relativity theory [6,7]:

$$
D^{2} \partial^{l} \partial_{l} \psi+i D \partial_{t} \psi-\frac{V}{2} \psi=0
$$

results from Equation (C7) for movements of the complex system structural units on Peano-type curves, $D_{F}=2$ and the correspondence $\lambda \equiv D$, where $D$ is the coefficient of the fractal, the non-fractal transition from scale relativity theory [6,7].

\section{References}

1. Chen, F. Introduction to Complex System Physics, 2nd ed.; Springer: New York, NY, USA, 1994.

2. Morozov, I. Introduction to Complex System Dynamics; CRC Press: Boca Raton, FL, USA, 2012.

3. Dimitriu, D.G.; Aflori, M.; Ivan, L.M.; Ioniţă, C.; Schrittwieser, R.W. Common physical mechanism for concentric and non-concentric multiple double layers in plasma. Plasma Phys. Control. Fusion 2007, 49, doi:10.1088/0741-3335/49/3/004.

4. Agop, M.; Dimitriu, D.G.; Niculescu, O.; Poll, E.; Radu, V. Experimental and theoretical evidence for the chaotic dynamics of complex structures. Phys. Scr. 2013, 87, 045501.

5. Dimitriu, D.G.; Aflori, M.; Ivan, L.M.; Radu, V.; Poll, E.; Agop, M. Experimental and theoretical investigations of plasma multiple double layers and their evolution to chaos. Plasma Sour. Sci. Technol. 2013, 22, 035007.

6. Nottale, L. Fractal Space-Time and Microphysics: Towards a Theory of Scale Relativity; World Scientific: Singapore, Singapore, 1993.

7. Nottale, L. Scale-Relativity and Fractal Space-Time; World Scientific: Singapore, Singapore, 2011.

8. Munceleanu, V.; Păun, V.P.; Casian-Botez, I.; Agop, M. The microscopic-macroscopic scale transformation through a chaos scenario in the fractal space-time theory. Int. J. Bifurc. Chaos 2011, 21, 603-618.

9. Agop, M.; Magop, D. New considerations in fractal space-time. In Classification and Application of Fractals: New Research; Mitchell, E.W., Murray, S.R., Eds.; Nova Science Publishers: Hauppauge, NY, USA, 2012; pp. 131-159.

10. Gouyet, J.-F. Physique et Structures Fractales; Masson: Paris, France, 1992. (In French)

11. Agop, M.; Gavriluț, A.; Crumpei, G.; Doroftei, B. Informational non-differentiable entropy and uncertainty relations in complex systems. Entropy 2014, 16, 6042-6058.

12. Agop, M.; Gavriluţ, A.; Stefan, G.; Doroftei, B. Implications of Non-differentiable entropy on a space-time manifold. Entropy 2015, 17, 2184-2197.

13. Mandelbrot, B. The Fractal Geometry of Nature; W.H. Freeman and Company: San Francisco, CA, USA, 1982.

14. Lifshiëtìs, E.M.; Landau, L.D. Fluid Mechanics; Pergamon Press: Oxford, UK, 1987.

15. Mazilu, N.; Agop, M. Skyrmions: A Great Finishing Touch to Classical Newtonian Philosophy; Nova Science Publishers: Hauppauge, NY, USA, 2012.

16. Mazilu, N.; Agop, M.; Axinte, C.I.; Radu, E.; Jarcău, M.; Gârţu, M.; Răuţ, M.; Pricop, M.; Boicu, M.; Mihăileanu, D.; et al. A Newtonian message for quantization. Phys. Essays 2014, 27, 202-214.

17. Mazilu, N.; Agop, M.; Boicu, M.; Mihăileanu, D.; Pricop, M.; Gaţu, I.; Dezideriu, D.; Ghizdovăţ, V. The geometry of heavenly matter formations. Phys. Essays 2015, 28, 120-127.

18. Agop, M.; Gavriluţ, A.; Ştefan, G. SL(2, R) invariance of the Kepler type motions and Shannon informational entropy. Uncertainty relations through the constant value of the Onicescu informational energy. Rep. Math. Phys. 2015, 75, 101-112.

19. Agop, M.; Gavriluţ, A. Some generalized physical models through homographic group. Rep. Math. Phys. 2015, 76, 231-246.

20. Agop, M.; Gavriluţ, A.; Rezuş, E. Implications of Onicescu's informational energy in some fundamental physical models. Int. J. Mod. Phys. B 2015, 29, 1550045.

21. Mercheş, I.; Agop, M. Differentiability and Fractality in Dynamics of Physical Sciences; World Scientific: Singapore, Singapore, 2015.

22. Éfimov, N. Géométrie Supérieure; Mir Publishers: Moscow, Russia, 1981. (In French) 
23. Barbilian, D. Opera Didactica, Volume I; Didactic and Pedagogical Publishing House: Bucharest, Romania, 1968. (In Romanian)

24. Barbilian, D. Opera Didactica, Volume II; Didactic and Pedagogical Publishing House: Bucharest, Romania, 1971. (In Romanian)

25. Barbilian, D. Opera Didactica, Volume III; Didactic and Pedagogical Publishing House: Bucharest, Romania, 1974. (In Romanian)

26. Mihăileanu, N. Complements of Geometry: Analytical, Projective and Differential; Didactic and Pedagogical Publishing House: Bucharest, Romania, 1972. (In Romanian)

(C) 2016 by the authors; licensee MDPI, Basel, Switzerland. This article is an open access article distributed under the terms and conditions of the Creative Commons Attribution (CC-BY) license (http:/ / creativecommons.org/licenses/by/4.0/). 